\title{
Modeling the mutual relationship between the circadian clock and inflammation response
}

\author{
Nikolay Podkolodnyy \\ ICM\&MG SB RAS \\ Novosibirsk, Russia \\ pnl@bionet.nsc.ru
}

\author{
Natalya Tverdokheb \\ Systems biology dapartment \\ ICG SB RAS \\ Novosibirsk, Russia \\ nata@bionet.nsc.ru
}

\author{
Olga Podkolodnaya \\ Systems biology dapartment \\ ICG SB RAS \\ Novosibirsk, Russia \\ opodkol@bionet.nsc.ru
}

\begin{abstract}
The computer model combining the circadian oscillator (CO) with the NAMPT/NAD+/SIRT1 system and the immune and inflammatory response system through the transcription factor NF- $\mathrm{KB}$ was developed. Modeling showed that an age-related decrease in SIRT1 activity might be one of the causes of malfunction of $\mathrm{CO}$, which may also lead to disturbances in the circadian rhythms of the body as a whole. It is likely that age-related changes in SIRT1 activity caused by NAD+ depletion can contribute to the separation of metabolic processes and circadian rhythm, which in itself can play an important role in the aging process. The simulation also showed that a decrease in the activity of Sirt1 with age leads to an increase in the activity of $\mathrm{NF}-\mathrm{kB}$, which can contribute to the aggravation of the course of inflammatory diseases.
\end{abstract}

Keywords - circadian clock, SIRT1, NAD+, aging, mathematical modeling, inflamation

\section{Motivation and Goals}

The main function of the circadian clock is to synchronize the cell biological processes to adapt it to rhythmically changing external influences. Circadian disruption can cause various diseases and disorders that manifest during a person's life.

It is generally accepted that circadian clocks function due to transcriptional-translational feedbacks between genes / proteins of the core of the circadian oscillator $(\mathrm{CO})$, which includes seven groups of genes - Clock (gene Clock and its homolog Npas2), Bmal (genes Bmall and Bmal2), Per (genes Per1, Per2 and Per3), Cry (genes Cryl and Cry2), CK1 (genes CKle and CK1d), Rev-erb (genes Rev-erbo and Rev-erb $\beta$ ) and Ror (genes Ror $\alpha, \operatorname{Ror} \beta$ and Ror $\gamma$ ), which form oscillations with a period close to 24 hours.

Studies of the last decade allow us to take a fresh look at the possible connections between the circadian rhythm and other functional systems of the body.

In particular, new data on the involvement of NAD+ dependent histone deacetylase SIRT1 in the integration of circadian rhythm and metabolic regulation pathways allow us to define the new NAD+ function as a "metabolic oscillator" [1]. SIRT1 is one of the metabolic sensors that respond to various external stimuli.

Its deacetylating activity affects a wide range of substrates, among which there are both structural and regulatory proteins, so changes in its activity affect a wide variety of processes at both the cellular and systemic levels. Its effects may be associated with the survival and differentiation of cells, the development of the body and lifespan, metabolism, inflammation, the development of neurodegenerative diseases and cancer [2,3].

SIRT1 targets in the CO core can be the BMAL1 gene and protein, PER2 protein, as well as chromatin in the region of CLOCK:BMAL1 binding sites. The enzymatic activity of SIRT1 depends on the level of NAD+, which in this case acts as a co-substrate for this deacetylase. The bottleneck link in the biosynthesis and recycling of NAD+ is the enzyme NAMPT. Nampt gene expression is regulated by the transcription factor CLOCK:BMAL1 and exhibits circadian oscillations at the level of mRNA and protein. In accordance with this, the level of NAD + and, therefore, the activity of SIRT1 also changes with a 24-hour period.

Thus, the functioning of the NAMPT/NAD+/SIRT1 system is closely related to the cell's $\mathrm{CO}$ and we can be assumed that the interaction of $\mathrm{CO}$ with many biological systems can be carried out through this system. In particular, now it has been shown that the transcription factor NF- $\kappa B$ (the main regulator of the immune and inflammatory response) is an important regulator of the circadian clock which necessary to maintain the circadian rhythm both at the molecular and behavioral levels. The CO elements interacting with $\mathrm{NF}-\kappa \mathrm{B}$ are the heterodimeric transcription factor CLOCK:BMAL1, its subunits CLOCK and BMAL1, as well as histone deacetylase SIRT1.

The aim of the work was to develop a computer model combining the CO with the NAMPT/NAD+/SIRT1 system, which ensures the interaction of the circadian clock system with the immune and inflammatory response system through the transcription factor $\mathrm{NF}-\kappa \mathrm{B}$.

\section{Background and Methods}

We reconstructed the circadian rhythm regulation gene network of mammals based on the annotation of 280 scientific publications and information from databases which includes 55 genes, 126 proteins, 16 non-protein substances, 439 reactions and regulatory events.

The reconstructed gene network describes the interactions of a number of $\mathrm{CO}$ elements including heterodimeric transcription factor CLOCK:BMAL1, its subunits CLOCK and BMAL1, as well as histone deacetylase SIRT1 with transcription factor NF- $\kappa \mathrm{B}$, which is the main transcriptional regulator of the immune and inflammatory response systems.

The transcription factor NF- $\mathrm{BB}$ is the main transcriptional regulator of the immune and inflammatory response system. In most cell types, NF- $\mathrm{KB}$ is predominantly represented by the p65:p50 heterodimer [4]. The activity of the transcription factor NF- $\kappa \mathrm{B}$ is regulated at the level of post-translational modification of its subunits. One of these modifications is acetylation of $\mathrm{p} 65$ with CLOCK acetyltransferase, a subunit of the main transcriptional regulator of the cell $\mathrm{CO}$ CLOCK:BMAL1 and deacylation of the same site with SIRT1 deacetylase. 


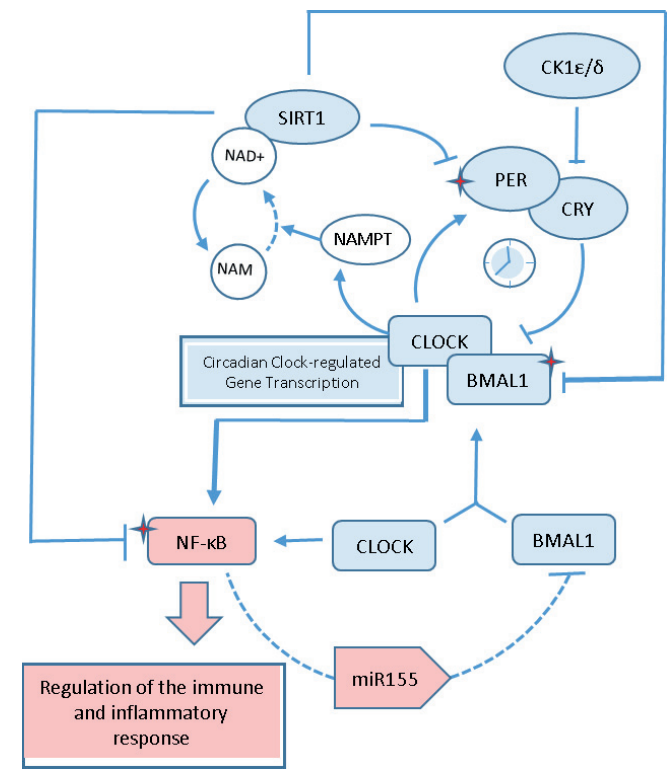

Fig. 1. Scheme of interaction of the circadian oscillator $(\mathrm{CO})$ with the NAMPT/NAD+/SIRT1 system and the immune and inflammatory response system through the transcription factor $\mathrm{NF}-\kappa \mathrm{B}$

High Bmall expression suppresses the activating effect of CLOCK on the transcriptional activity of NF- $\kappa \mathrm{B}$ and is manifested as a decrease in the expression of NF- $\kappa \mathrm{B}-$ regulated genes $[4,5]$. It is likely that BMAL1 and p65 compete for interaction with CLOCK, which can lead to disruption of the circadian oscillator mechanism of the cell under conditions of increased activity in response to inflammatory stimuli (Fig. 1).

These starting points were used to build a model for the interaction of the circadian rhythm regulation system with the immune system and the inflammatory process.

\section{Results and Discussion}

We modified and expanded the mathematical model of mammalian $\mathrm{CO}$ created by Kim and Forger [6], adding the following subsystems to it:

- The NAMPT / NAD+ / SIRT1 pathway.

- The pathway of enhancing the degradation of PER2 after its deacetylation with the SIRT1 enzyme.

- The pathway of the effect of SIRT1 on the transcription of the gene Bmall and inhibition of the CLOCK:BMAL1 function associated with the E-box through histone deacylation.

- The pathway of interaction of CO with the transcription factor NF- $\kappa \mathrm{B}$, which is the main transcriptional regulator of the immune and inflammatory response systems.

The extended model is presented as a system of 186 ordinary differential equations (186 variables and 81 parameters).

The dynamics of changes in the main components of the extended model are consistent with the experimental data that were used in the original model of Kim and Forger [6].

Modeling while minimizing the activity of SIRT1 showed that the expression of $\mathrm{CO}$ genes keeps rhythm, however, the amplitude of oscillations and the general expression level of a number of genes, including Per2, Bmall and Rev-erb, decreased, which is consistent with experimental data [7]. In addition, a decrease in the oscillation amplitude of the BMAL1 protein level and the CLOCK:BMAL1 complex, the main positive regulator of $\mathrm{CO}$ transcription, was noted.

Based on experimental data on the age-related dynamics of SIRT1 activity $[7,8]$ we included age as a parameter in the model. This allowed us to model age-related changes in the behavior of CO. Modeling revealed a decrease in the expression of mRNA of the Bmal1, Per2, Rev-Erba genes and a prolongation of the oscillation period with age, which has experimental confirmation.

Our simulation also showed that the activity of NF-kB has a pronounced circadian character with a maximum at 6 o'clock in the morning. An analysis of the mortality rate of mice depending on the dose and time of endotoxin injection was performed in [4] where it was shown that the maximum mortality rate of a mouse from a pathological reaction is observed for endotoxin, administered at 6 a.m. - the time of maximum activity of NF-kB detected as a result of modeling.

Thus, our results indicate that an age-related decrease in SIRT1 activity may be one of the causes of impaired functioning of $\mathrm{CO}$, which may also lead to disturbances in the circadian rhythms of the body as a whole. The simulation also showed that a decrease in the activity of Sirt1 with age leads to an increase in the activity of NF-kB, which can contribute to the aggravation of the course of inflammatory diseases.

\section{ACKNOWLEDGMENT}

This work was supported by project 0324-2019-0040-C01 from the Russian State Budget.

\section{REFERENCES}

[1] S. Imai, "Clocks in the NAD World: NAD as a metabolic oscillator for the regulation of metabolism and aging". Biochim Biophys Acta. 2010, Vol.1804(8), pp.1584-1590.

[2] S. Masri, "Sirtuin-dependent clock control: new advances in metabolism, aging and cancer". Curr Opin Clin Nutr Metab Care. 2015 Nov. Vol.18(6), pp.521-527.

[3] S.H. Cho, J.A. Chen, F. Sayed, M.E. Ward, F. Gao, T.A. Nguyen, G. Krabbe, P.D. Sohn, I. Lo, S. Minami, N. Devidze, Y. Zhou, G. Coppola, L. Gan, "SIRT1 deficiency in microglia contributes to cognitive decline in aging and neurodegeneration via epigenetic regulation of IL-1 $\beta$ ”. J Neurosci. 2015 Jan 14;35(2), pp.807-818.

[4] M.L. Spengler, K.K. Kuropatwinski, M. Comas, A.V. Gasparian, N. Fedtsova, A.S. Gleiberman, I.I. Gitlin, N.M. Artemicheva, K.A. Deluca, A.V. Gudkov, M.P. Antoch, "Core circadian protein CLOCK is a positive regulator of NF-kB-mediated transcription". Proc Natl Acad Sci U S A. 2012 Sep 11;109(37), pp.E2457-E2465.

[5] A.M. Curtis, C.T. Fagundes, G. Yang, E.M. Palsson-McDermott, P. Wochal, A.F. McGettrick, N.H. Foley, J.O. Early, L. Chen, H. Zhang, C. Xue, S.S. Geiger, K. Hokamp, M.P. Reilly, A.N. Coogan, E. Vigorito, G.A. FitzGerald, L.A. O'Neill, "Circadian control of innate immunity in macrophages by miR-155 targeting Bmall”. Proc Natl Acad Sci U S A. 2015 Jun 9;112(23), pp. 7231-7236.

[6] J.K. Kim, D.B. Forger, "A mechanism for robust circadian timekeeping via stoichiometric balance". Mol Syst Biol. 2012, Vol.8, p. 630 .

[7] H.C. Chang, L. Guarente, "SIRT1 mediates central circadian control in the SCN by a mechanism that decays with aging". Cell. 2013 Jun 20;153(7), pp.1448-1460.

[8] R.H. Wang, T. Zhao, K. Cui, G. Hu, Q. Chen, W. Chen, X-W. Wang, A. Soto-Gutierrez, K. Zhao, C-X. Deng, "Negative reciprocal regulation between Sirt1 and Per2 modulates the circadian clock and aging”. Sci Rep. Vol.6, 28633 (2016). 